\title{
Insights from Public Health Researchers into the Digital Transformation of an Educational Lifestyle Course
}

\author{
William BEVENS a , Kathleen GRAY a, Tracey WEILAND a and George JELINEK ${ }^{\text {a }}$ \\ ${ }^{a}$ The University of Melbourne, IOS Press, Australia
}

\begin{abstract}
The last decade has seen an explosion in the uptake of digital health interventions (DHI) to address complex chronic diseases. This is particularly true in the case of multiple sclerosis where those living with the disease are increasingly seeking adjuvant treatment options such as lifestyle management. This paper seeks to give perspectives and insights from public health researchers that have engaged in the digital transformation process of a face-to-face lifestyle management educational program. There is a dearth of information regarding the digital transformation of lifestyle educational programs and this is particularly true for programs directed at chronic diseases. A large body of work exists from higher education, an area that has undergone rapid digital transformation of its work, and much can be derived from this field. There is also a well-established field of design methodologies and frameworks available to researchers seeking to design, develop and implement DHIs. This paper provides a practical overview of the synthesis between digital transformation processes in higher education and the application of an existing development framework for DHIs. By describing this process, we hope to fill an existing gap within the literature that will provide a valuable tool for future researchers.
\end{abstract}

Keywords. digital health interventions, digital transformation, chronic disease, lifestyle education

\section{Introduction}

Multiple sclerosis (MS) is a chronic, degenerative, autoimmune disease that affects the central nervous system in around 2.3 million people worldwide. The disease course of MS is highly variable (1); the prognosis for those newly diagnosed provides little certainty in how significantly and rapidly the disease may progress. As there is no cure currently available, treating the underlying disease course as well as the associated perturbations is an important concept for MS. There now exists a large body of evidence implicating the role of environmental and lifestyle factors into the health outcomes for people with MS (PwMS) $(2,3)$. These factors include diet, exercise, smoking, vitamin D and sun exposure and stress. Ultimately, the strong epidemiological basis for some of these factors have yet to be translated into gold standard randomised control trial data, particularly not in combination. This prevents mainstream acceptance of tailored lifestyle recommendations and therefore future research should focus its efforts on high-quality randomised control trials (RCTs) in order to traverse this gap.

The most comprehensive evidence for a multimodal lifestyle intervention comes from the STOP-MS study run by our team. This longitudinal study tracks a large cohort 
of PwMS that have undertaken a 5-day in-person live-in education program aimed at educating participants on the Overcoming Multiple Sclerosis (OMS) 7-step program that provides best-practice information on diet, physical activity, smoking cessation, vitamin $\mathrm{D}$ and stress reduction. Results from this longitudinal study have spanned 5 years of follow-up, showing an improvement and sustainment of a range of important health outcomes for PwMS (4-6). However, significant limitations impede the conducting of RCTs testing face-to-face multimodal lifestyle interventions, which include prohibitive costs, accessibility concerns, maintenance of behavioural change and recruitment, including issues around randomisation and blinding.

Through the use of digital health technologies, many of these limitations can be overcome. Therefore, our team is undertaking the digital transformation of the existing face-to-face OMS lifestyle educational course into a web-based format. Currently, while there are a number of valuable resources for researchers to develop DHIs, they often lack the generalisability required for public health researchers generally and specifically, for the digital transformation process required for our purposes. Further, the process of translating a pre-existing face-to-face course aimed at behavioural change is not a process that is well described in the literature and research teams themselves are often not experienced or lack the skills to appropriately apply these resources. There are key considerations that are not present when designing DHIs without prior material and therefore this needs to be explored further.

This paper will provide insights into the digital transformation process for behavioural change interventions with particular emphasis on the experiences of public health researchers. Due to the increasing utilisation of DHIs and the dearth of information regarding a digital transformation process, we believe it necessary to communicate our experiences and insights into this design and development process, which this paper seeks to do.

\section{Design Methodologies}

Despite the wealth of information available to researchers to aid in the design stages of DHIs with many groups having advanced their own models or theories to aid in a methodological approach to development, these remain underutilised by research teams developing DHIs outside the health informatics space. In a recent unpublished review on DHIs for PwMS, we found that only 6 out of 17 studies included reported any description of a process design or methodology in the development of their intervention. While often it is difficult to ascertain whether there exists a genuine lack of the use of any framework or whether it is a lack of reporting, authors were surveyed who confirmed these data. Significantly, this made it difficult to replicate previous methods that showed success in these studies, which is a problem more broadly for DHIs (7). This poses an important question: what is required to fill this gap between the established field of DHI design methodologies and the explosion in utilisation of DHIs to address health issues?

\subsection{Research Silos}

In our experience, this problem relates to an issue of 'research siloing' whereby the skills of an entire discipline, that being digital health and health informatics, were not present within our research group. This is likely to be the case in most public health research groups and is definitely the case within our department and school. In particular, public 
health informatics research is not highly visible in Australia (8), and this requires researchers to make connections into discplines previously unexplored. In our case, this was straightforward due to working within a large, well-resourced institution, with a School of Computing and Information Systems, E-research group and vast array of online resources. This may not be the case for many other institutions and therefore should be considered an important part of any institution's future plans. Overall, while the idea of interdisciplinary collaboration is not novel, the value of a contemporary discipline such as health informatics needs to be appreciated by more traditional disciplines such as public health.

This may be explained by the attitudes towards digital health by health researchers themselves. Academics may be perceived as lacking technological skills, which may translate to their dismissal of its overall value in academia (9). Further, cost-effectiveness, rather than effectiveness on the health outcomes of interest has been described as the main driver for adopting digital heath technologies (10), whereby priority is placed on rapid developments at the expense for thorough planning.

Efforts are ongoing to resolve the issue of research 'silos' by recognising the diversity of skills necessary to tackle macro-problems. This is none more evident than in the current effort to tackle the global coronavirus pandemic. Emphasis should be placed on continuing the work in integrating health informatics within disciplines, including public health.

\section{Digital Transformation}

There is likely to be a difference in approach required between a digital transformation project such as ours, and a project whereby a DHI is designed from scratch. This is an unexplored area within the literature; while there exist interventions that have been 'digitised', the experiences taken from this process or the underlying methodology have not been well-reported. It is clear that many existing interventions will undergo a digitisation in the future and therefore, explorations of this process are vital.

For the purposes of our project, this transformation process involved augmenting the initial stages of the design process. As is recognised across the majority of digital health development frameworks, the initial stages of this process are to be characterised by analysis of the needs of end-users and the context in which the intervention will be implemented. For our purposes, the framework we selected was the CeHReS roadmap (11) due to its amalgamation of participatory design and persuasive design elements, described in a way that was understandable to our research team.

For a digital transformation project, this initial step will vary slightly in its intended use because the problem statement is likely already identified. Further. that intervention may have already undergone extensive quantitative and qualitative testing to uncover both the mechanism of action of participant experiences with the intervention. This was the case with our intervention as we had more than a decade of delivery to many hundreds of PwMS and multiple quantitative and qualitative publications. One example is that our research team already recognised the necessity of accessibility features due to potential for participants within our cohort to present with physical and/or cognitive impairments. What remained for us in terms of a digital transformation process was to understand the problems associated with the current format of delivery, the solutions that may emerge from a digital platform and to address the limitations associated with this transformation. 


\subsection{Advisory Group}

This process took place utilising an advisory group of PwMS from the community. This group comprised those who had previous experience with the face-to-face program and other associated resources whilst others had heard of the program but had very little exposure to it. This group, through semi-structured group conversations, provided insight into a wide range of important aspects of the pre-existing course and elements that should be considered for a digital course. In particular, two important elements of the existing course were identified from these discussions as areas to which our team should pay close attention: peer support and experts delivering content. It was recognised that the transformation of these two key elements into a digital environment was a key aspect to the success of this project. The process of elucidating key components of a pre-existing behavioural change intervention by both the research team and end-users via the contextual inquiry stage of design facilitates this process.

These two themes were then interrogated as to how they exerted their intended effect on the outcome of interest in the existing course, and then how they may operate within the digital space. In terms of our project, this meant exploring what was successful about the experts delivering educational content and the peer-support elements of the course, which in turn drove behavioural changes that led to improved health previously described.

\subsection{Expert Educational Content}

Formal educational content process within the face-to-face course was delivered via seminar style presentations led by experts (physicians, researchers or dieticians) in their chosen discipline. Briefly, these seminars covered topics on diet, physical activity, stress reduction, sun exposure and vitamin $\mathrm{D}$, medication and family prevention. The format of these seminars followed an informal structure of 'why' and 'how': firstly, clinical and research data was presented on a topic and illustrated visually with figures and images; secondly, information was provided on how to implement the recommendations according to the research data previously presented.

The practical, physical task of transforming the content will occur between researchers and developers, be they researchers as well or industry professionals. For our purposes, once the types and frequency of multimedia elements to exist in the platform was decided, content templates were generated by the development team, and these templates were then filled by members of the research team. This methodical approach of content implementation was a skill our research team was not familiar with and one that was introduced by the industry professional team.

\subsection{Peer-support}

The benefits of peer-support for chronic diseases within digital environments has been previously described and contain a range of synchronous and asynchronous approaches (12). The benefits and limitations of any particular approach needed to be considered by the research team. In our case, these benefits and negatives were informed both on our prior experiences with the face-to-face course and the opinions raised within the advisory group. In the face-to-face course, participants would learn from others in an experiential, peer-support manner, which was unstructured and could occur any time participants interacted. It is important to note that because the face-to-face course is a 5-day residential course, interaction occurs consistently as participants go about their daily 
activities. This is not feasible in a modular, online educational course and therefore useful alternatives needed to be considered.

The advisory group was split on the implementation of a synchronous chat system but ultimately agreed that there were alternatives outside the platform that may be preferable. The "anonymity" of a DHI would give participants pause to use a synchronous tool to communicate with each other due to the perceived inability to develop emotional connections in the online course format. Participants indicated that they had prior experience engaging with forums, particularly those familiar with OMS program who had engaged with the OMS forums, and that would be a comfortable medium for peer-to-peer interaction for an online course.

With this information, it was decided that the peer-support component (experiential content) would be facilitated using an asynchronous forum system with light moderation. This method of peer-support is a large deviation from how this occurs within the faceto-face program for three important reasons: it occurs in a virtual space as opposed to physical; it is asynchronous; and participants are aware of moderation and that their communication is visible to the wider groups. It is not clear what the outcomes of these differences will be however future feasibility testing will clarify this.

\subsection{Development Team}

Choosing a development team is a difficult decision, as the majority of researchers will lack the understanding of the skills and expertise required to undertake a project as large and important as many DHIs. Additionally, while the field of DHIs is relatively new as an academic discipline, it is novel as a venture for small businesses also, who may seek to enter into this market with little experience in this field. For all these reasons, our team initially found ourselves engaged with a contractor that was unable to deliver upon our intended design. It is unclear how common this is as the design and development process is often not articulated fully and appropriately within the literature. Fortunately, through a series of networking processes, we located a team who had previously interacted with the facilitators of the residential course and with the general skills and experience to engage in a task such as ours. Interdisciplinary collaboration will give research teams the resources and skills to both find, vet and collaborate with development teams.

\section{Summary}

Based upon the issues and insights raised in this paper, we propose some key recommendations for research teams and institutions:

1. Ensuring research groups are multidisciplinary. An appreciation of the complexity involved in the design, development and administering of DHIs is required by public health researchers, which will ideally lead to the incorporation of health informatics researchers into their groups.

2. Provide centralised lists of recommended developers. This is critical in ensuring that researchers are engaging those that are best equipped to develop DHIs alongside researchers. As it is often difficult for researchers with little knowledge of DHIs to recognise what skills are needed, a list such as this would be invaluable.

3. The development of digital transformation methodologies. Much of this process can be informed from existing design methodologies and frameworks dedicated 
to digital health however, there remain elements specific to transformational projects that are unaccounted for in these models.

Ultimately, we believe implementing these key recommendations will allow researchers greater access to better resources that will ensure DHIs are appropriately designed, developed and therefore, adequately evaluated.

\section{References}

[1] Tsang BK, Macdonell R. Multiple sclerosis- diagnosis, management and prognosis. Australian family physician. 2011;40(12):948-55.

[2] Jakimovski D, Guan Y, Ramanathan M, Weinstock-Guttman B, Zivadinov R. Lifestyle-based modifiable risk factors in multiple sclerosis: review of experimental and clinical findings. Neurodegener Dis Manag. 2019;9(3):149-72.

[3] Belbasis L, Bellou V, Evangelou E, Ioannidis JP, Tzoulaki I. Environmental risk factors and multiple sclerosis: an umbrella review of systematic reviews and meta-analyses. Lancet Neurol. 2015;14(3):26373.

[4] Hadgkiss EJ, Jelinek GA, Weiland TJ, Rumbold G, Mackinlay CA, Gutbrod S, et al. Health-related quality of life outcomes at 1 and 5 years after a residential retreat promoting lifestyle modification for people with multiple sclerosis. Neurol Sci. 2013;34(2):187-95.

[5] Li MP, Jelinek GA, Weiland TJ, Mackinlay CA, Dye S, Gawler I. Effect of a residential retreat promoting lifestyle modifications on health-related quality of life in people with multiple sclerosis. Quality in primary care. 2010;18(6):379-89.

[6] Marck CH, De Livera AM, Brown CR, Neate SL, Taylor KL, Weiland TJ, et al. Health outcomes and adherence to a healthy lifestyle after a multimodal intervention in people with multiple sclerosis: Three year follow-up. PLoS One. 2018;13(5):e0197759.

[7] Coiera E, Ammenwerth E, Georgiou A, Magrabi F. Does health informatics have a replication crisis? Journal of the American Medical Informatics Association. 2018;25(8):963-8.

[8] Gray K, Martin Sanchez F. Public Health Informatics in Australia and around the World. Public Health Informatics in Australia and around the World. Telehealth and Mobile Health: Taylor; 2015.

[9] Sucala M, Nilsen W, Muench F. Building partnerships: a pilot study of stakeholders' attitudes on technology disruption in behavioral health delivery and research. Translational Behavioral Medicine. 2017;7(4):854-60.

[10] Topooco N, Riper H, Araya R, Berking M, Brunn M, Chevreul K, et al. Attitudes towards digital treatment for depression: A European stakeholder survey. Internet Interventions. 2017;8:1-9.

[11] van Gemert-Pijnen JE, Nijland N, van Limburg M, Ossebaard HC, Kelders SM, Eysenbach G, et al. A Holistic Framework to Improve the Uptake and Impact of eHealth Technologies. J Med Internet Res. 2011;13(4):e111.

[12] Merolli M, Gray K, Martin Sanchez F. Health outcomes and related effects of using social media in chronic disease management: a literature review and analysis of affordances. J Biomed Inform. 2013;46(6):957-69. 